\title{
Design of Infrared Detection Device for Pesticide Residues in Crop Leaves
}

\author{
Qiaoyu Guo, a , Shuhang Luan, ${ }^{2, b}$, Jinpeng Song ${ }^{1, c}$, \\ Yan Wang ${ }^{1, d}$, Xumeng Zhang ${ }^{1,}$ and Limin Shao ${ }^{1, f^{*}}$ \\ ${ }^{1}$ College of Mechanical and Electrical Engineering, Hebei Agricultural University, Baoding 071001, \\ China \\ ${ }^{2}$ College of Modern Science and Technology, Hebei Agricultural University, Baoding 071001, China \\ a3174895523@qq.com, b80739801@qq.com, c1360164194@qq.com, d1853715694@qq.com, \\ 'real_doris@163.com, 'shaolm@126.com
}

\section{Keywords: Near-infrared; Crop leaves; Pesticide detection lossless}

\begin{abstract}
Rapid and efficient detection of pesticide residues in crops is a major concern of current food safety control. At present, the detection method of pesticide residues has the shortcomings of the cumbersome process of the early treatment of the crop samples, the consumption of reagents, and the long time consuming, which can not meet the needs of modern rapid and green detection. The advantage of infrared detection technology in nondestructive testing has broad application prospects in the field of crop detection. In this paper, the basic principle, application characteristics of near-infrared technology and the design principle of infrared detection device for pesticide residues in crop leaves are summarized. The device was used to detect chlorpyrifos as an example, using the near-infrared detection principle, the characteristic range of the reflected infrared wavelength of the pesticide, and the detection of pesticide residues in the crop leaves by the silicon photocell. The leaves of crops are detected by infrared and after reflection through the filter, and the silicon photoelectric cell converts light signals into electrical signals, thus reflecting the degree of pesticide residues. The device can detect the pesticide residues in the growing crop leaves quickly and lossless, avoid the lag problem of pesticide detection, improve the process of crop pesticide treatment, improve the precision of pesticide spraying, and can timely reflect the agricultural information and guide the agricultural production.
\end{abstract}

\section{Introduction}

Pesticide is a kind of mixture and its preparations, mainly used to prevent, eliminate or control the hazard of agriculture, forestry disease, insect, grass and other harmful organisms, regulating plant growth, in order to achieve the purpose of promoting crop yield. Pesticide is a kind of special chemical, it can prevent agroforestry pests, also can cause harm to human and animals [1]. In the process of spraying pesticide, the concentration of pesticides and the amount of spraying are often controlled, so the pesticide residue is often overused, and even the pesticide is sprayed repeatedly to achieve the purpose of killing. And the excessive pesticide residual on plant leaves, will have great harm to human body, therefore in the process of crop planting need to timely detection of plant leaves pesticide residue [2], in order to achieve the purpose of the controllable spray pesticide in a timely manner. This device is designed to take pesticide chlorpyrifos as an example. It is used to detect the pesticide residues. Chlorpyrifos is also known as chlorpyrifos standard or chlorpyriphos brodan. The pesticide with stomach poison, tag, triple play, fumigation of rice, wheat, cotton, fruit, vegetables, tea tree on a variety of chewing and sucking mouthparts pests have good pest control effect [3]. Traditional pesticide residue detection methods have a fruit and vegetable crops, liquid chromatography, gas chromatography, liquid mass usage, temperament coupon usage, enzyme-linked immunoassay and so on, and although the test method of high sensitivity, good accuracy, but the crop of the early stage of the process operation trival, can real-time detect pesticide residues in the process of crop growth, it is hard to meet the current rapid batch high efficiency, low cost and so on pesticide residues detection requirements. The near-infrared detection technology has the advantages of nondestructive, simple operation and quick response, which can be applied to real-time detection of crop pesticide residues. 


\section{Near-Infrared Detection Technology Principle}

Near-infrared detection technology is a new analysis and detection technology with many characteristics such as nondestructive, rapid and low cost, which can easily detect the status of pesticide residues in crops [4]. The near-infrared wavelength (near-infrared band) range $780 \sim 3000 \mathrm{~nm}$, which is an electromagnetic wave between visible light and far infrared light, produces rich spectral information on the frequency doubling and frequency absorption of interatomic vibration within the group molecules such as $\mathrm{O}-\mathrm{H}, \mathrm{C}-\mathrm{H}$ and $\mathrm{N}-\mathrm{H}$. The frequency of near-infrared light and the frequency of the vibration of the pesticide are different; the near-infrared light of this frequency will not be absorbed. When the crops are irradiated with near-infrared light, the crop leaves can selectively absorb the near-infrared light of different frequencies. The near-infrared light will weaken in some wavelength range after the crop leaves, and the reflected infrared light is carried the characteristic information of the composition and structure of the pesticide. The absorption of the light density of the reflected light and the special range of the wavelength light can reflect the residual degree of pesticide on the crop leaves, so as to realize the detection of pesticide residues in the crop leaves by near-infrared detection technology [5]. The near red detection technology has the characteristics of nondestructive, easy to operate, low detection cost, rapid reaction and environmental protection, and can be used to detect pesticide residues on crop leaves [6-8].

\section{Device Design}

The infrared feature detection device for pesticide residues in crop leaves is adopted near-infrared detection technology. The device converts electrical signals into light signals. Infrared light irradiates the leaves of crops in darkrooms, and leaves reflect longer wavelengths of light. Through the filter, the silicon photocell receives the reflected light and converts the light signal into an electrical signal[9-10]. Pass to stm32 MCU. The larger the residues of the pesticides, the more the silicon cells receive the light and the greater the voltage. The infrared feature detection device for pesticide residues in crop leaves is composed of switch handle, test chamber, open door, circuit room and display screen. The structure diagram of the infrared feature detection device for pesticide residues in crop leaves is shown in Fig.1.

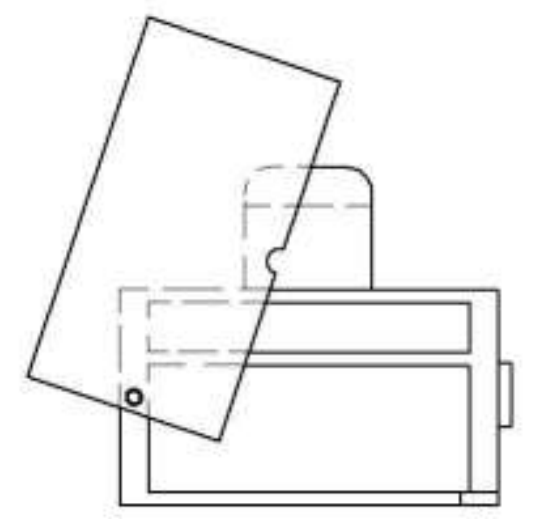

Figure 1. Infrared detection device structure

The switch handle contains a battery. Press the switch, can open the screen and near-infrared light source, and thus start the device detection work. The detection box contains infrared light, filter and silicon photocell. Infrared light wavelength of $808 \mathrm{~nm}$ infrared light, light on plant leaves, plant leaves on pesticide reflecting characteristic wavelengths of light, filter can filter the light wavelength range of 700 $\sim 700 \mathrm{~nm}$. The silicon photocell receives only the light reflected from the pesticide. Therefore, the higher the concentration of pesticides, the stronger the reflected light, the stronger the optical signal received by the silicon cell. Silicon photocell acceptable between $290 \sim 1200 \mathrm{~nm}$ near-infrared light, therefore can be on the reflection characteristics of chlorpyrifos pesticide band of light in reaction, the corresponding light signal into electrical signal, passed to the STM32 MCU, to judge the degree of pesticide residues. 
The device door allows the device detection box to form a dark chamber to prevent the external light from interfering with the detection result. The door of the device can be rotated around the axis of rotation, and a semicircle groove at the bottom of the door is used to place the stems of plants so as to detect pesticides on crop leaves in the growing state. To protect internal dark environment test kits, on the outside of the semicircle groove with a layer of black elastic layer, tight encirclement of plant stem, thus reducing the outside world to measure the light, to improve the accuracy of detection.

Circuit room, located above the detection room, is used for placing circuit boards, STM32 single chip microcomputer, voltage reducing module and other electronic control devices. The control diagram of single chip microcomputer is shown in Fig.2.

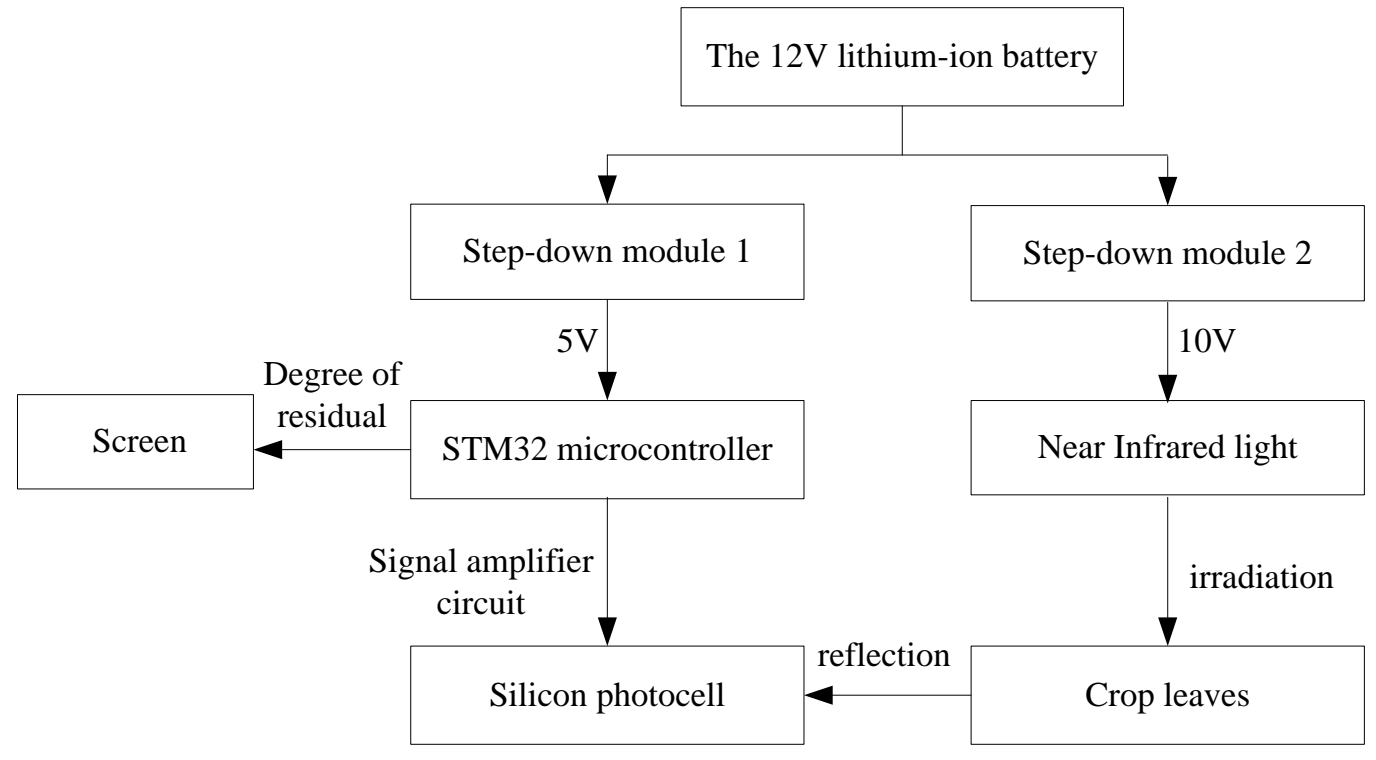

Figure 2. System chart of control

The display screen is used to show the results of pesticide residue detection, and the flow chart of the device is shown in Fig.3.

\section{Experimental Results}

The device is designed to detect chlorpyrifos by mid infrared detection technology. When pesticide residues on crop leaves are $>3.875 \%$, the screen will show strong pesticide residues. When pesticide residues on crop leaves are at $0.775 \%-3.875 \%$, the screen will show mid-range pesticide residues. When pesticide residues in crop leaves are less than $0.775 \%$, the device display will show that pesticide residues are weak.

\section{Conclusion}

The infrared detection device of crop leaves is used to detect the degree of pesticide residues in crop leaves by using the principle of infrared detection and the principle of the characteristic range of the reflected wavelength of the pesticide. It can be tested in the condition of plant growth with real-time and rapidity, and can be controlled by pesticide residues. The device has a simple structure and a wide range of crops. It is suitable for testing cotton, tobacco, soybean and other crops. Because the device has no damage to plants, it is fast and low cost, so it has wide market application prospects. 


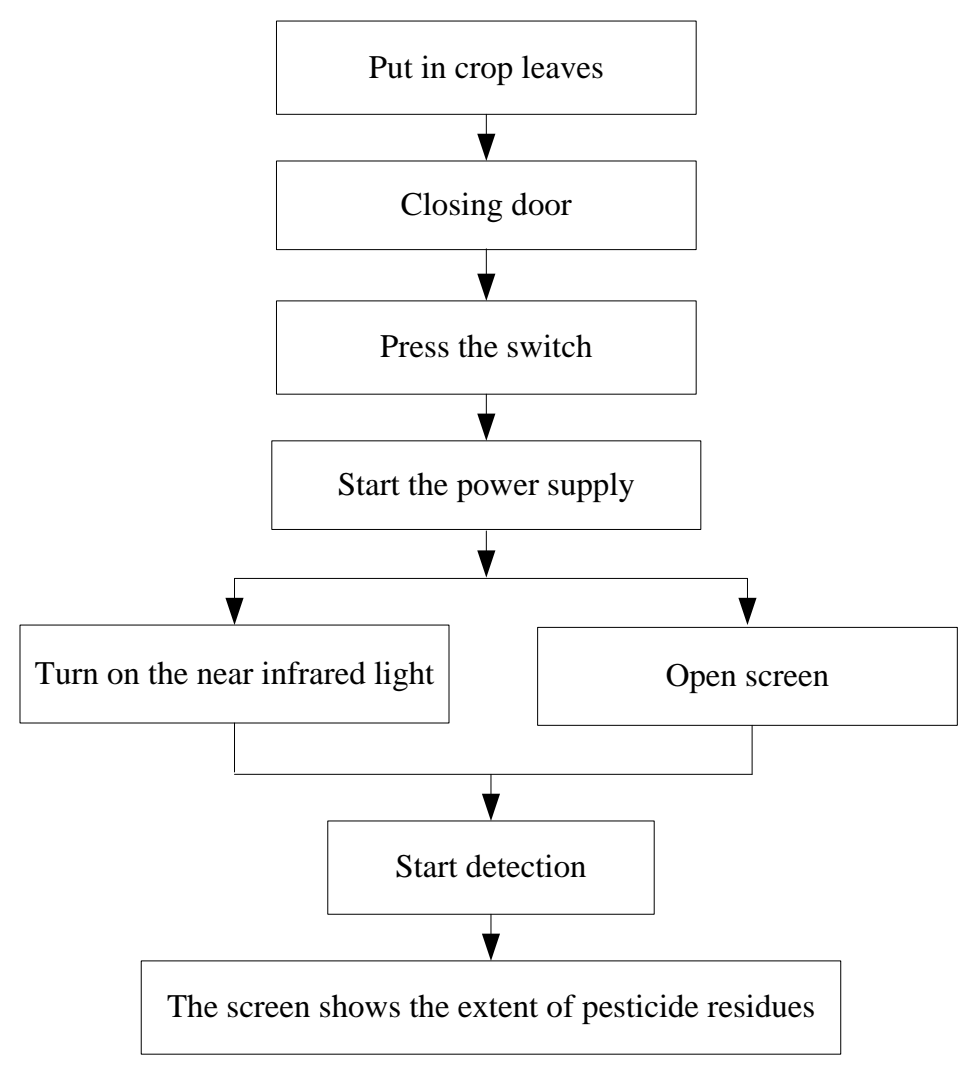

Figure 3. Flow chart

\section{Acknowledgements}

This study was supported by the Undergraduate Innovation and Entrepreneurship Training Program of Hebei Agricultural University (201810086050) and the Key Research and Development Program of Hebei Province (18227209D, 18227209D-1).

\section{References}

[1] P.Z. Li, W.J. Chen, Y.P. Luo: Modern Agrochemicals, Vol.8 (2009) No.3, p.38.

[2] X.Q. Qu, R.M. Hua, J.Y. Wu, et al.: Journal of Anhui Agriculture Science, Vol.35 (2007) No.36, p.11743.

[3] D.H. Wu: Spectroscopy and Spectral Analysis, Vol.28 (2008) No.7, p.1530.

[4] G. Li, Z. Zhao, R. Liu, et al.: Spectroscopy and Spectral Analysis, Vol.31 (2011) No.9, p.2412.

[5] W.Y. Chen. Pesticide Production and Synthesis (Chemical Industry Press, China 2000).

[6] X.Y. Niu, D.J. Gong, Y.W. Wang, et al.: Modern Food Science and Technology, Vol.30 (2014) No.12, p.130.

[7] Z.L. Zhao, D.J. Gong, Y.W. Wang, et al.: Spectroscopy and Spectral Analysis, Vol. 36 (2016) No.7, p.2089.

[8] X.Y. Niu, Z.L. Zhao, K.J.Jia, et al.: Food Chemistry, Vol. 133 (2012) No.2, p.592.

[9] G. Tang, K.D. Tian, X.Z. Song, et al.: Spectrochimica Acta Part A Molecular \& Biomolecular Spectroscopy, Vol.121 (2014) No.5, p.678.

[10] C.Y. Gu, B.R. Xiang, J.P. Xu: Spectrochim Acta A Mol Biomol Spectrosc, Vol.97 (2012) No.6, p.594. 